\title{
Induction shimming: A new shimming concept for superconductive undulators
}

\author{
D. Wollmann, ${ }^{*}$ A. Bernhard, P. Peiffer, and T. Baumbach \\ University of Karlsruhe, Kaiserstrasse 12, 76131 Karlsruhe, Germany \\ R. Rossmanith \\ Forschungszentrum Karlsruhe, P.O. Box 3640, 76021 Karlsruhe, Germany
}

(Received 11 June 2008; published 17 October 2008)

\begin{abstract}
Undulators are the most advanced sources for the generation of synchrotron radiation. The photons generated by a single electron add up coherently along the electron trajectory. In order to do so, the oscillatory motion of the electron has to be in phase with the emitted photons along the whole undulator. Small magnetic errors can cause unwanted destructive interferences. In standard permanent magnet undulators, the magnetic errors are reduced by applying shimming techniques. Superconductive undulators have higher magnetic fields than permanent magnet undulators but shimming is more complex. In this paper it is shown that coupled superconductive loops installed along the surface of the superconductive undulator coil can significantly reduce the destructive effect of the field errors. This new idea might allow the building of undulators with a superior field quality.
\end{abstract}

DOI: 10.1103/PhysRevSTAB.11.100702

PACS numbers: $84.71 . \mathrm{Ba}, 41.20 . \mathrm{Gz}, 41.75 . \mathrm{Ht}, 29.20 . \mathrm{dk}$

\section{INTRODUCTION}

Undulators are the most effective sources for brilliant $\mathrm{x}$ rays in storage rings. Up to now undulators were mainly made from permanent magnets. This technique was optimized over many years. The maximum magnetic field strength of permanent magnet undulators is limited by the material properties of the permanent magnets $[1,2]$. Two new concepts tried to overcome these limitations.

The first one was the development of in-vacuum permanent magnet undulators with a smaller magnetic gap and higher on-axis field strengths. By cooling the in-vacuum devices to temperatures between $150 \mathrm{~K}$ and liquid nitrogen temperature, the on-axis field can be increased further and at the same time the risk of radiation damage is reduced [3-5].

A second concept consisted of replacing the permanent magnets by superconductive wires. After first experiments in the 1970's [6,7], the development was resumed in the early 1990's [8,9] and several intermediate steps were followed by the installation of a 100 period superconductive undulator with a period length of $14 \mathrm{~mm}$ in the storage ring ANKA in April $2005[10,11]$. This device made it possible to study the properties of the emitted radiation [12], the beam heat load [13], the effect of the undulator on the orbit [14], the field errors, and the development of correction schemes for field errors $[15,16]$.

In an undulator the electrons continuously emit white light into a narrow cone around the forward direction ( $z$ axis). These cones overlap and the photons emitted by a single electron interfere. Because of this interference the undulator emits a line spectrum along the $z$ axis:

*daniel.wollmann@physik.uni-karlsruhe.de

$$
\lambda_{L}=\frac{\lambda_{u}}{2 k \gamma^{2}}\left(1+\frac{K^{2}}{2}\right)
$$

$\lambda_{L}$ is the wavelength of the photons, $\lambda_{u}$ is the period length of the undulator, $\gamma$ the relative beam energy, and $k$ the harmonic number of the emitted radiation $(k=$ $1,3,5, \ldots)$. The deflection parameter $K$ is $K=0.0934$. $\lambda_{u}[\mathrm{~mm}] \cdot \tilde{B}[\mathrm{~T}]$, with $\tilde{B}$ the amplitude of the magnetic field on the beam axis.

In order to obtain the maximum brilliance, the photons must add up constructively. A phase slip between the electron and the photon would cause a line broadening and intensity reduction of the emitted lines. A measure for the deviation between electron and photon phase is the so-called phase error. For an overview see [17,18].

Field errors in permanent magnet and hybrid (permanent magnets and poles) undulators can be due to a number of factors, e.g. variation of permanent magnet strength, magnetization errors, material inhomogeneities, and mechanical tolerances $[1,18]$.

In superconductive undulators the distance of the poles and wire bundles to the beam axis and the period length vary around the nominal values. Therefore, by ensuring tight mechanical tolerances and by iteratively applying mechanical measurements and mechanical corrections (readjusting poles, grinding, etc.), it is possible to reduce the expected phase errors significantly. Other possible sources of field errors are variations in pole material and persistent currents. Electrical shimming concepts have been proposed and verified for single undulator periods $[19,20]$. These have subsequently been extended to whole undulators $[16,21,22]$.

For both types of undulators shimming is an iterative and time consuming process of measuring, applying shims or 
shim coil, and verifying the improved field quality. This provided the stimulation to think about concepts for superconductive undulators where field errors are compensated automatically without additional steps of shimming [23].

\section{INDUCTION SHIMMING}

The induction-shimming concept for superconductive undulators is based on Faraday's law of induction,

$$
\oint_{C} \tilde{\mathbf{E}} d \vec{l}=-\frac{d}{d t} \int_{S} \tilde{\mathbf{B}} d \vec{A}
$$

where $\tilde{\mathbf{B}}$ is the magnetic flux density over the area $S$ with the contour $C, \tilde{\mathbf{E}}$ is the electrical field strength, and $d \vec{A}$ is the surface element. The line integral of the electric field strength $\tilde{\mathbf{E}}$ along $C$ is equal to the negative time derivative of the integral of the magnetic flux density $\tilde{\mathbf{B}}$ over the area $S$, which is confined by the contour $C$.

Using an ideal conductor along the contour, for instance a superconductive closed loop, Eq. (2) is reduced to

$$
0=\frac{d}{d t} \int_{S} \tilde{\mathbf{B}} d \vec{A}
$$

In other words, a change of the magnetic flux through the closed loop is compensated by the magnetic flux produced by the induced current.

\section{A. One period with closed loop}

The idea is to use closed superconductive loops for phase-error compensation. These closed loops must be arranged in such a way that for an ideal undulator the magnetic flux enclosed by one loop is equal to zero. This is the case when the loop covers integer multiples of undulator periods.

In the following a superconductive closed loop is considered, which is installed on the undulator surface and covers a full period (see Fig. 1). Further on, it is assumed that the magnetic flux in each half period is different. For

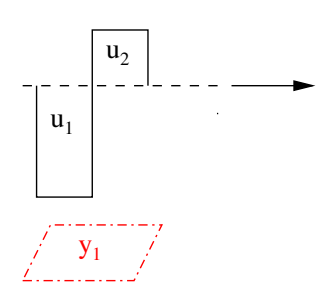

a)

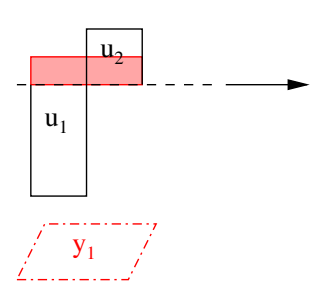

b)

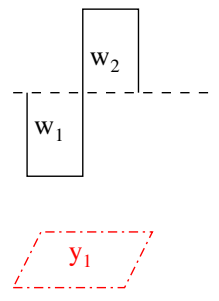

c)
FIG. 1. (Color) Influence of a superconductive closed loop installed at the undulator surface. The loop covers a single undulator period. Without the induced current in the loop (a) the magnetic flux in the two half periods is different. For the sake of simplicity it is assumed that the flux has a rectangular shape. When the loop is installed, the induced current produces a correction flux [red (b)] and equalizes the field strength according to Eq. (3) (c). the sake of simplicity it is assumed that the flux in each half period has a rectangular shape. The magnetic flux enclosed by the superconductive loop is not zero [see Fig. 1(a)].

The magnetic flux in the first and second half period $u_{1}$ and $u_{2}$ and the flux $y_{1}$ produced by the loop superpose to zero:

$$
y_{1}+u_{1}+u_{2}=0 .
$$

The flux $w_{1}$ and $w_{2}$ in each half period is changed to

$$
\begin{aligned}
& w_{1}=u_{1}+\frac{1}{2} y_{1} \\
& w_{2}=u_{2}+\frac{1}{2} y_{1} .
\end{aligned}
$$

Equations (4)-(6) yield

$$
\begin{aligned}
w_{1}+w_{2} & =0 \\
\rightarrow w_{2}=-w_{1} & =w .
\end{aligned}
$$

Equations (5) and (6) can be rewritten as follows:

$$
w=-u_{1}-\frac{1}{2} y_{1} \quad w=u_{2}+\frac{1}{2} y_{1},
$$

and solved for $w$,

$$
w=\frac{-u_{1}+u_{2}}{2} .
$$

The current in the loop equalizes the field strength according to Eq. (3). This is shown in Fig. 1(c).

\section{B. Generalization for $\boldsymbol{n}$ overlapping closed loops}

In the following, induction shimming with superconductive closed loops is extended to $n$ closed loops.

The system is shown in Fig. 2, with the magnetic flux $u_{n}$ in the $n$th half period $(n=1,2,3, \ldots)$, the correction flux $y_{m}$ produced by the closed loop $m(m=1,2,3, \ldots, n-1)$, and the resulting fluxes $w_{n}$.

The set of equations for Faraday's law can be written as

$$
\begin{aligned}
y_{1}+u_{1}+u_{2}+\frac{1}{2} y_{2} & =0 \\
y_{2}+u_{2}+u_{3}+\frac{1}{2} y_{1}+\frac{1}{2} y_{3} & =0 \\
& \vdots \\
y_{n-1}+u_{n-1}+u_{n}+\frac{1}{2} y_{n-2}+\frac{1}{2} y_{n} & =0 \\
y_{n}+u_{n}+u_{n+1}+\frac{1}{2} y_{n-1} & =0 .
\end{aligned}
$$
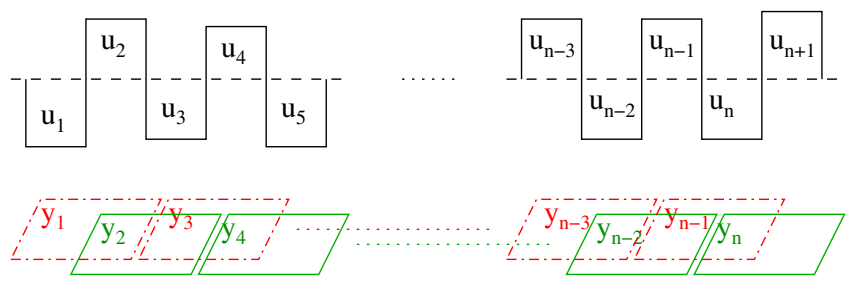

FIG. 2. (Color) Rectangular flux with $n+1$ half periods and $n$ overlapping closed loops. 
The magnetic flux in each half period is

$$
\begin{aligned}
w_{1} & =u_{1}+\frac{1}{2} y_{1} \\
w_{2} & =u_{2}+\frac{1}{2} y_{1}+\frac{1}{2} y_{2} \\
& \vdots \\
w_{n} & =u_{n}+\frac{1}{2} y_{n-1}+\frac{1}{2} y_{n} \\
w_{n+1} & =u_{n+1}+\frac{1}{2} y_{n} .
\end{aligned}
$$

Substituting these equations into the closed-loop equations (9) gives

$$
\begin{aligned}
& w_{1}+w_{2}=0 \\
& w_{2}+w_{3}=0 \\
& \vdots \\
& w_{n-1}+w_{n}=0 \\
& w_{n}+w_{n+1}=0,
\end{aligned}
$$

which is equal to

$$
w= \pm w_{1}=\mp w_{2}=\cdots= \pm w_{n}=\mp w_{n+1} .
$$

The magnetic flux in each half period becomes

$$
\begin{aligned}
w & = \pm u_{1} \pm \frac{1}{2} y_{1} \\
w & =\mp u_{2} \mp \frac{1}{2} y_{1} \mp \frac{1}{2} y_{2} \\
& \vdots \\
w & = \pm u_{n} \pm \frac{1}{2} y_{n-1} \pm \frac{1}{2} y_{n} \\
w & =\mp u_{n+1} \mp \frac{1}{2} y_{n},
\end{aligned}
$$

which can be solved for $w$ :

$$
w=\frac{ \pm u_{1} \mp u_{2} \pm \cdots \pm u_{n} \mp u_{n+1}}{n+1} .
$$

As with the one loop system described before, the system of $n$ overlapping closed loops adjusts the absolute values of the magnetic flux in each half period to the same level.

\section{GENERALIZATION FOR BIOT-SAVART CLOSED LOOPS}

In the following the concept is extended to sinusoidal fields and the field produced by the current in the loops is described by the law of Biot-Savart.

For the sake of simplicity the closed loops are considered to consist of ideally superconducting long straight wires perpendicular to the beam direction ( $z$ axis). The loop parts parallel to the e-beam direction can be neglected due to their large distance from the e-beam.

\section{A. Faraday's law for overlapping closed loops in a long undulator}

Faraday's law of induction [see Eq. (2)] for one closed loop can be written as

$$
\dot{I}=-\frac{1}{L} \dot{\Phi},
$$

where $\dot{\Phi}$ is the time derivative of the magnetic flux through the closed loop, $L$ is the self-inductance of the loop, and $\dot{I}$ the time derivative of the induced current. For an induction-shimming scheme with overlapping closed loops, the coupling between the loops also has to be considered. The coupling between loop $i$ and loop $j$ with a mutual inductance $M_{i j}$ is defined by

$$
\dot{I}_{i}=M_{i j} \dot{I}_{j}
$$

Combining Eqs. (13) and (14) and solving for $\dot{\Phi}$ gives

$$
\dot{\Phi}_{i}=L\left(\sum_{j \neq i} M_{i j} \dot{I}_{j}-\dot{I}_{i}\right)
$$

When the current in the undulator main coil is turned on at the time $t_{0}$, the flux through the closed loops is changed from zero to a certain value and a current $I$ is induced. Integration of Eq. (15) yields

$$
\Phi_{i}=L\left(\sum_{j \neq i} M_{i j} I_{j}-I_{i}\right) .
$$

The self-inductance $L$ and the mutual inductances $M_{i j}$ are defined by the geometrical arrangement and the design of the closed loops.

\section{B. Biot-Savart's law for overlapping closed loops in a long undulator}

In a real superconductive undulator, a set of overlapping closed loops will be mounted in the way shown in Fig. 3. One possibility would be to use high temperature superconductor loops sputtered on a substrate and structured by applying lithographic techniques. The achievable geometric accuracy with this technique is in the order of $10 \mathrm{~nm}$.

The undulator consists of $N$ periods and the shimming system of $2 N-1$ closed loops. The loops are numbered from the entrance of the undulator $(1,2, \ldots, 2 N-1)$. Errors of the undulator magnetic field induce correction currents in the closed loops. The main field and the correction fields superpose in the beam plane. For this arrangement of superconductive closed loops, the selfinductance and the mutual inductances will be determined in the following with the help of Biot-Savart's law.

The magnitude of the magnetic flux density $\tilde{\mathbf{B}}$ at a point $P$ at the distance $R=\sqrt{z^{2}+y^{2}}$ caused by a current $I$ in a long straight wire, running parallel to the $x$ axis, is given by [24] 


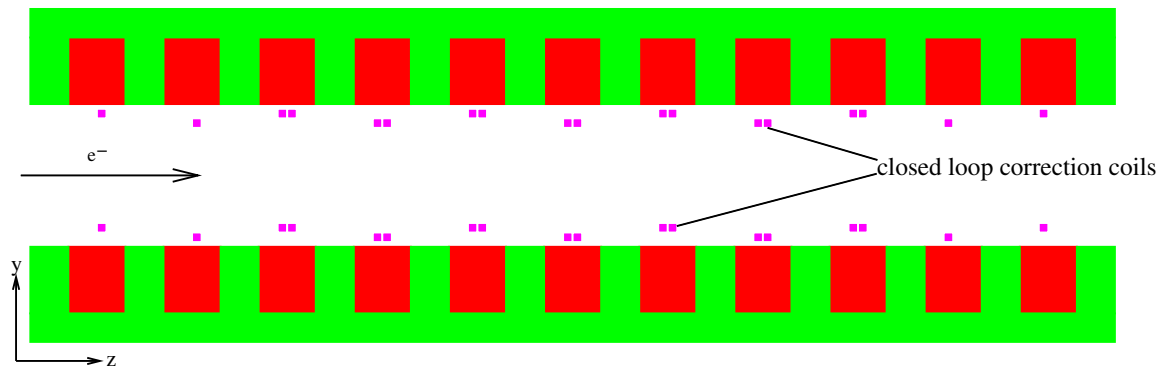

FIG. 3. (Color) Cross section of an undulator with the main coils (green, iron; red, superconductive wire bundles) and overlapping closed loops for correction (magenta) placed close to the main coils.

$$
|\tilde{\mathbf{B}}|=\frac{\mu_{0}}{4 \pi} I R \int_{-\infty}^{\infty} \frac{d l}{\left(R^{2}+l^{2}\right)^{3 / 2}}=\frac{\mu_{0}}{2 \pi} \frac{I}{R}
$$

$\mu_{0}=4 \pi \times 10^{-7} \frac{\mathrm{Vs}}{\mathrm{Am}}$ is the magnetic permeability in vacuum. The coordinate system is defined in Fig. 3.

With $\cos \alpha=\frac{z}{R}$ and the above definition of $R$, the $y$ component of the magnetic flux density at a point can be calculated as

$$
B_{y}(y, z)=|\tilde{\mathbf{B}}| \cos \alpha=\frac{\mu_{0} I}{2 \pi} \frac{z}{z^{2}+y^{2}},
$$

where $z$ and $y$ are the distances from the center of the conductor to point $P$. The conductor has the dimensions $w_{c c} \times h_{c c}$.

Treating this as a two-dimensional problem, the magnetic flux produced by the loop shown in Fig. 4 is

$$
\begin{aligned}
\Phi & =2 \int_{w_{c c} / 2}^{\lambda_{u}-(3 / 2) w_{c c}} B_{y}\left(z^{\prime}\right) d z^{\prime}=2 \frac{\mu_{0} I}{2 \pi} \int_{w_{c c} / 2}^{\lambda_{u}-(3 / 2) w_{c c}} \frac{1}{z^{\prime}} d z^{\prime} \\
& =2 \frac{\mu_{0}}{4 \pi} I\left(\ln \frac{\left(\lambda_{u}-\frac{3}{2} w_{c c}\right)^{2}}{\left(\frac{w_{c c}}{2}\right)^{2}}\right)=-L I .
\end{aligned}
$$

The mutual inductances $M_{i j}$ for $i \neq j$ and $i, j=$ $1 \ldots(2 N-1)$ can be calculated with the help of Fig. 5 . Defining

$$
a_{k}=L M_{i, j}
$$

with $k=|j-i|$ and

$$
\xi_{k}= \begin{cases}1 & \text { if } k \text { is odd } \\ 0 & \text { if } k \text { is even }\end{cases}
$$

$a_{k}$ is given by

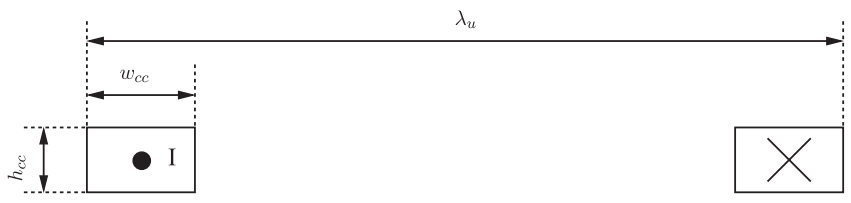

FIG. 4. One closed loop made out of two long straight wires.

$$
\begin{aligned}
a_{k}= & \frac{\mu_{0}}{2 \pi} \int_{(k / 2) \lambda_{u}+(1 / 2) w_{\mathrm{cc}}}^{[(k / 2)+1] \lambda_{u}-(3 / 2) w_{\mathrm{cc}}} \frac{z^{\prime}}{z^{\prime 2}+\left(\xi_{k} h_{\mathrm{cc}}\right)^{2}} d z^{\prime} \\
& -\frac{\mu_{0}}{2 \pi} \int_{[(k / 2)-1] \lambda_{u}+(3 / 2) w_{\mathrm{cc}}}^{(k / 2) \lambda_{u}-(1 / 2) w_{\mathrm{cc}}} \frac{z^{\prime}}{z^{\prime 2}+\left(\xi_{k} h_{\mathrm{cc}}\right)^{2}} d z^{\prime},
\end{aligned}
$$

or

$$
\begin{aligned}
a_{k}= & \frac{\mu_{0}}{4 \pi}\left(\ln \left[\frac{\left[\left(\frac{k}{2}+1\right) \lambda_{u}-\frac{3}{2} w_{\mathrm{cc}}\right]^{2}+\left(\xi_{k} h_{\mathrm{cc}}\right)^{2}}{\left(\frac{k}{2} \lambda_{u}+\frac{1}{2} w_{\mathrm{cc}}\right)^{2}+\left(\xi_{k} h_{\mathrm{cc}}\right)^{2}}\right]\right) \\
& -\frac{\mu_{0}}{4 \pi}\left(\ln \left[\frac{\left(\frac{k}{2} \lambda_{u}-\frac{1}{2} w_{\mathrm{cc}}\right)^{2}+\left(\xi_{k} h_{\mathrm{cc}}\right)^{2}}{\left[\left(\frac{k}{2}-1\right) \lambda_{u}+\frac{3}{2} w_{\mathrm{cc}}\right]^{2}+\left(\xi_{k} h_{\mathrm{cc}}\right)^{2}}\right]\right) .
\end{aligned}
$$

The integrals were solved analytically [25].

Equation (16) becomes

$$
\left(\begin{array}{c}
\Phi_{1} \\
\Phi_{2} \\
\vdots \\
\Phi_{2 N-1}
\end{array}\right)=-M_{\mathrm{cc}}\left(\begin{array}{c}
I_{1} \\
I_{2} \\
\vdots \\
I_{2 N-1}
\end{array}\right),
$$

with the symmetrical matrix

$$
M_{\mathrm{cc}}=\left(\begin{array}{ccccc}
L & a_{1} & a_{2} & \cdots & a_{2 N-1} \\
a_{1} & & & \ddots & \vdots \\
a_{2} & & \ddots & & a_{2} \\
\vdots & \ddots & & & a_{1} \\
a_{2 N-1} & \cdots & a_{2} & a_{1} & L
\end{array}\right) .
$$

The resulting magnetic field along the beam axis can be written in the form

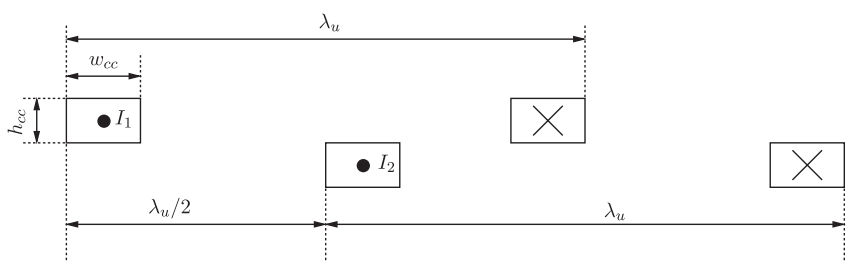

FIG. 5. Two overlapping closed loops with induced currents $I_{1}$ and $I_{2}$. 


$$
B_{y}(z)=B_{y, \text { main }}(z)+\sum_{l=1}^{2 N-1} B_{y, l}(z),
$$

where $B_{y \text {,main }}(z)$ is the magnetic field along the beam axis generated by the undulator main coils and $B_{y, l}(z)$ is the field generated by the closed-loop $l$ [see Eq. (18)].

\section{SIMULATIONS}

In the following it is assumed that the magnetic field is sinusoidal. Amplitude and period length can be varied in each half period. The midplane of the superconductive closed loops is considered to be $1 \mathrm{~mm}$ away from the source (main coil) and $2.5 \mathrm{~mm}$ from the beam plane. The undisturbed field amplitude on axis is $1 \mathrm{~T}$.

\section{A. Correction of a single field error}

For a first simulation, a three period undulator has been modeled with a $10 \%$ too high second maximum. A single pole, which is closer to the beam than the others, would cause this error. The field plot along the beam axis with and without induction shimming is shown in Fig. 6. The second maximum was reduced by about $7 \%$ from 1.1 to $1.03 \mathrm{~T}$. The absolute values of the neighboring minima were increased to $1.025 \mathrm{~T}$ and the first maximum was increased to $1.015 \mathrm{~T}$. The changes in the third maximum and minimum were negligible. The error previously localized in one half period was distributed over two periods.

The comparison of the first field integrals, with and without induction shimming, is shown in Fig. 7. The final value of the first field integral was reduced by a factor of 2 and the sign changed.

Comparing these results with those obtained in Sec. II with rectangular fields shows that the method with coupled Biot-Savart loops is somewhat less effective. This is due to the fact that in the rectangular field model the field is

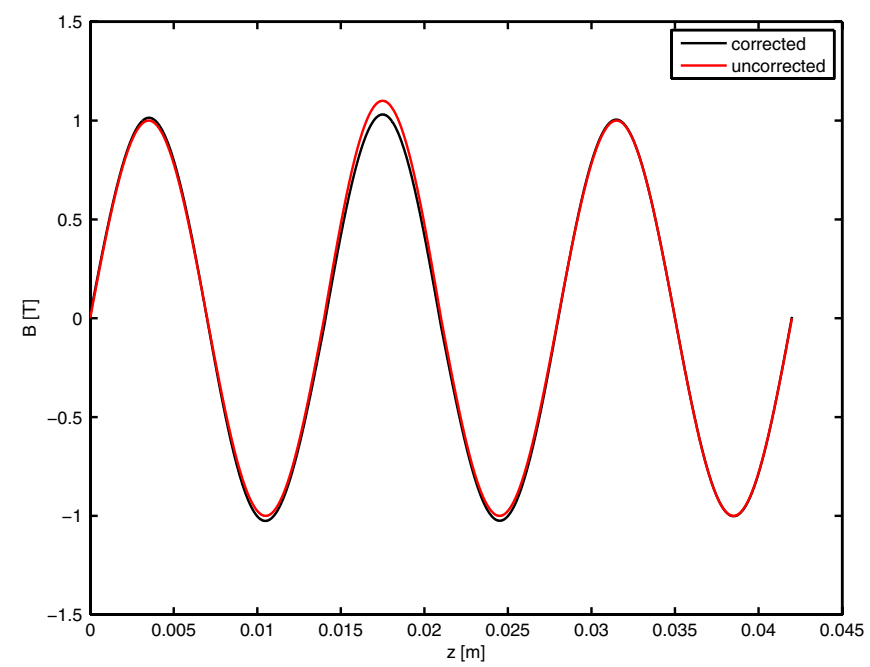

FIG. 6. (Color) Comparison of the magnetic field along the beam axis with (black) and without (red) induction shimming.

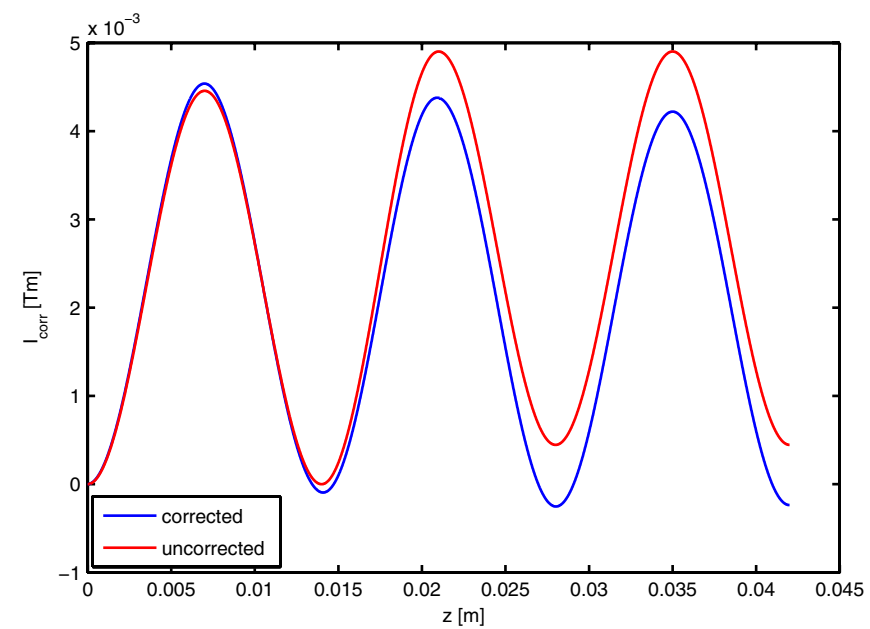

FIG. 7. (Color) Comparison of the first field integral along the beam axis with (blue) and without (red) induction shimming.

assumed to be confined inside the loop. But in reality the field generated by a loop expands to some extent into the space outside the loop and reduces the efficiency of the method. This reduction in efficiency can be partly compensated by using a different arrangement of the loops: the loops cover two or more periods instead of one.

\section{B. Monte Carlo simulations}

In a superconductive undulator, statistically distributed mechanical deviations are the main reasons for phase errors. Figures 8 and 9 show the phase-error distribution calculated for 1000 undulators with and without induction shimming. The undulators consist of 50 periods. Normally distributed variations of the wire-bundle $y$ positions (antisymmetric field deviation) and pole $y$ positions (single field

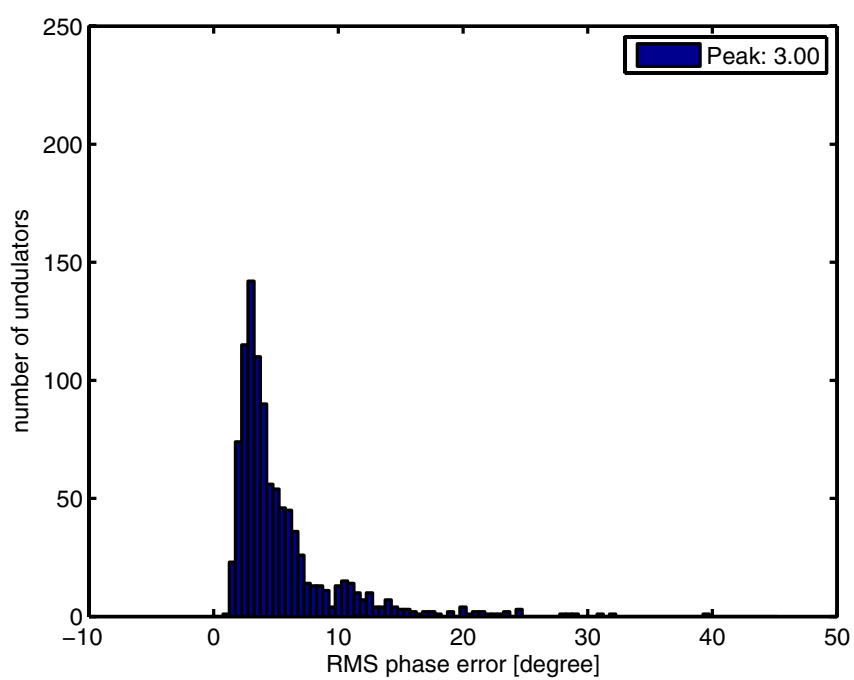

FIG. 8. (Color) Phase-error distribution for 1000 50-period undulators without induction shimming; $\sigma_{\Delta B / B \text {, coil }}=\sigma_{\Delta B / B \text {, pole }}=$ $3 \times 10^{-3}$. 


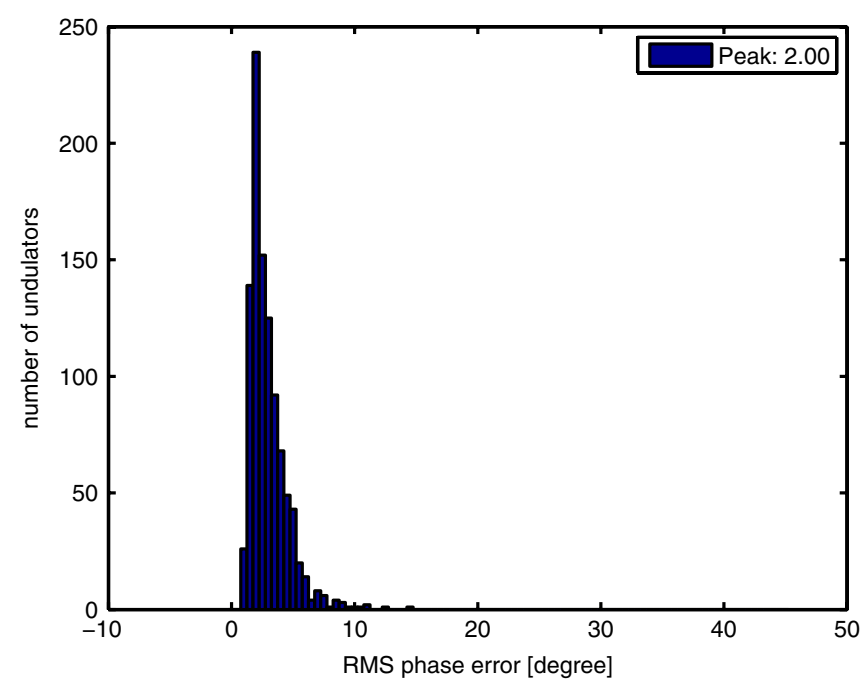

FIG. 9. (Color) Phase-error distribution for 1000 50-period undulators with induction shimming; $\sigma_{\Delta B / B \text {, coil }}=\sigma_{\Delta B / B \text {, pole }}=$ $3 \times 10^{-3}$.

distortion at one extremum), both with $\sigma_{\Delta B / B}=3 \times 10^{-3}$, were assumed.

In a second step the influence of a longitudinally misaligned induction-shimming system on the efficiency of the correction was examined. Therefore the whole induction-shimming loop arrangement was moved $0.5 \mathrm{~mm}$ against the zero crossings of the undulator main field. Figure 10 shows the phase-error distribution calculated for 1000 undulators with the misaligned inductionshimming system.

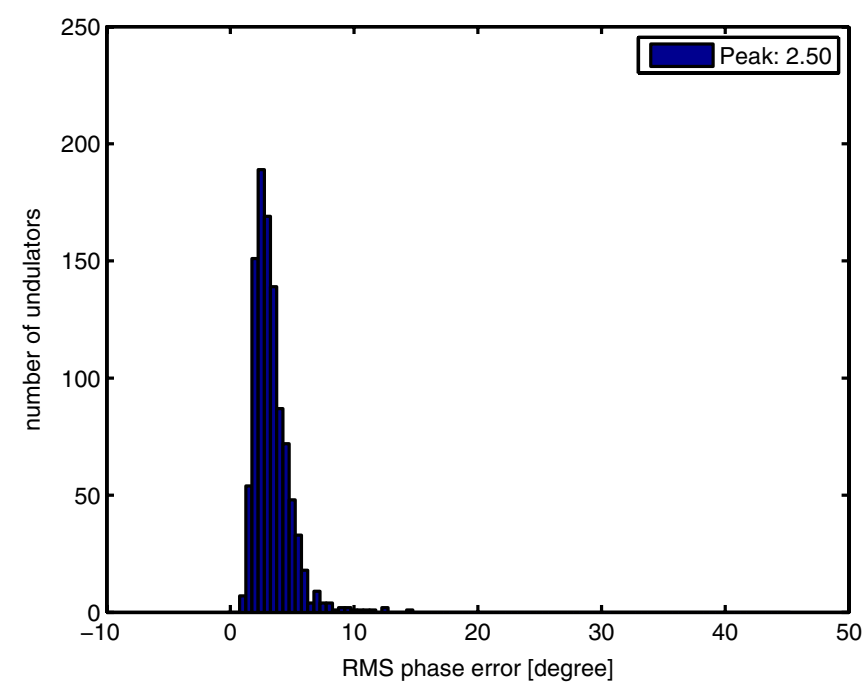

FIG. 10. (Color) Phase-error distribution for 1000 50-period undulators with a induction-shimming system misaligned by $0.5 \mathrm{~mm}$ against the zero crossings of the undulator main field; $\sigma_{\Delta B / B, \text { coil }}=\sigma_{\Delta B / B, \text { pole }}=3 \times 10^{-3}$.
TABLE I. Comparison of the confidence levels in the phaseerror distributions with and without induction shimming (ind. shim.).

\begin{tabular}{lccc}
\hline \hline & $\begin{array}{c}\text { Without } \\
\text { ind. shim. }\end{array}$ & $\begin{array}{c}\text { With } \\
\text { ind. shim. }\end{array}$ & $\begin{array}{c}\text { Misaligned } \\
\text { ind. shim. }\end{array}$ \\
\hline Peak of the distribution & $3.0^{\circ}$ & $2.0^{\circ}$ & $2.5^{\circ}$ \\
$50 \%$ level & $3.9^{\circ}$ & $2.5^{\circ}$ & $3.0^{\circ}$ \\
$99.7 \%$ level & $29^{\circ}$ & $11^{\circ}$ & $11.5^{\circ}$ \\
\hline \hline
\end{tabular}

The width of the phase-error distribution with induction shimming is significantly lower and the tail of the distribution is shorter. In case of a longitudinally misaligned induction-shimming system, the correction efficiency is reduced. The results are shown in Table I.

In addition, angular misalignments of the shimming system have to be considered. A lateral cant (roll) of the shim substrate will (i) reduce the flux enclosed by the loop and therefore reduce the correction current and (ii) generate additional horizontal field components.

A short estimation shows that these effects are small and therefore can be neglected in relation to the longitudinal misalignment: Assume a width of the substrate of $50 \mathrm{~mm}$ ( $x$ axis). The shim substrate is mounted at $x=-25 \mathrm{~mm}$ directly on the coil surface and at $x=+25 \mathrm{~mm}$ it is misplaced by $1 \mathrm{~mm}$ from the coil surface. The substrate has an angle $(d y / d x)$ relative to the coil surface of $\arctan (1 / 50)$. The projected loop area and therefore the enclosed flux is reduced to $\cos [\arctan (1 / 50)]=99.98 \%$ of the flux enclosed by a perfectly positioned loop. The induced current and the total correction field are reduced by about the same amount. The correction field has in first order an angle of $\arctan (1 / 50)$ to the $y$ axis. The vertical correction field has then a value of $\{\cos [\arctan (1 / 50)]\}^{2}=$ $99.96 \%$ and the additionally produced horizontal field component a value of $\sin [\arctan (1 / 50)]$. $\cos [\arctan (1 / 50)]=1.99 \%$ of the perfect vertical correction field.

Assuming that the shimming system can correct field errors not larger than $1 \%$ of the main coil field, the horizontal field component is negligible small. In addition, a misplacement by $1 \mathrm{~mm}$ seems to be a dramatic value: $0.1 \mathrm{~mm}$ is more likely the worst case. In this case the effects are reduced by a factor of 10 compared to the example given above.

Since the shim substrate is in general much longer than wide a rotation around the vertical axis ( $y$ axis) has an even smaller effect on the shimming efficiency than the one caused by a lateral cant.

The positioning of the induction-shimming system can be done iteratively. Therefore, misalignments can be reduced to an acceptable limit.

As discussed in the previous section the phase-error reduction could be further increased by using a different arrangement of the loops. 


\section{CONCLUSION}

A new shimming concept for superconductive undulators is described. The simulations showed that the phase error is reduced significantly.

The efficiency of the correction can be increased by using different loop arrangements: loops covering two or more periods instead of one.

[1] Undulators, Wigglers and their Applications, edited by H. Onuki and P. Elleaume (Taylor \& Francis, London, 2003), Vol. I, Chap. 5, pp. 148-213, ISBN 0-415-28040-0.

[2] J. Clarke, The Science and Technology of Undulators and Wigglers (Oxford Science Publication, New York, 2004).

[3] T. Hara, T. Tanaka, H. Kitamura, T. Bizen, X. Marechal, T. Seike, T. Kohda, and Y. Matsuura, Phys. Rev. ST Accel. Beams 7, 050702 (2004).

[4] C. Kitegi, J. Chavanne, D. Cognie, P. Elleaume, F. Revol, C. Penel, B. Plan, and M. Rossat, in Proceedings of the 10th European Particle Accelerator Conference, Edinburgh, Scotland, 2006 (EPS-AG, Edinburgh, Scotland, 2006), pp. 3559-3561.

[5] T. Bizen, X. Marechal, T. Seike, H. Kitamura, T. Hara, T. Tanaka, Y. Asano, D. Kim, and H. Lee, in Proceedings of the European Particle Accelerator Conference, Lucerne, 2004 (EPS-AG, Lucerne, 2004), pp. 2092-2094.

[6] Y. Farge, Appl. Opt. 19, 4021 (1980).

[7] A.S. Artamonov, L. M. Barkov, V.B. Baryshev, N. S. Bashtonoy, N.A. Vinokurov, E.S. Gluskin, G. A. Korniukhin, V.A. Kochubei, G. N. Kulipanov, N.A. Mezentsev, V.F. Pindiurin, A. N. Skrinsky, and V.M. Khorev, Nucl. Instrum. Methods 177, 239 (1980).

[8] H. Moser, B. Krevet, and H. Holzapfel, German Patent No. P 4101 094.9-33, 1991.

[9] I. Ben-Zvi, Z. Jiang, G. Ingold, and L. Wu, Nucl. Instrum. Methods Phys. Res., Sect. A 297, 301 (1990).

[10] A. Bernhard, M. Hagelstein, B. Kostka, R. Rossmanith, T. Schneider, M. Weisser, D. Wollmann, T. Baumbach, E. Steffens, and G. Gerlach, IEEE Trans. Appl. Supercond. 16, 1228 (2006).

[11] S. Casalbuoni, M. Hagelstein, B. Kostka, R. Rossmanith, M. Weisser, E. Steffens, A. Bernhard, D. Wollmann, and T. Baumbach, Phys. Rev. ST Accel. Beams 9, 010702 (2006).

[12] A. Bernhard, S. Casalbuoni, R. Frahm, B. Griesebock, U. Haake, M. Hagelstein, B. Kostka, Y.-L. Mathis, A.-S. Mueller, R. Rossmanith, F. Schoeck, E. Steffens, M.
Weisser, D. Wollmann, and T. Baumbach, IEEE Trans. Appl. Supercond. 17, 1235 (2007).

[13] S. Casalbuoni, A. Grau, M. Hagelstein, R. Rossmanith, F. Zimmermann, B. Kostka, E. Mashkina, E. Steffens, A. Bernhard, D. Wollmann, and T. Baumbach, Phys. Rev. ST Accel. Beams 10, 093202 (2007).

[14] D. Schoerling, Student thesis, Technische Universitaet Bergakademie Freiberg, Germany, 2007.

[15] Elena Mashkina, Andreas Grau, Theo Schneider, Axel Bernhard, Sara Casalbuoni, Michael Hagelstein, Barbara Kostka, Robert Rossmanith, Erhard Steffens, Daniel Wollmann, and Tilo Baumbach, J. Phys. Conf. Ser. 97, 012020 (2008).

[16] D. Wollmann, A. Bernhard, S. Casalbuoni, M. Hagelstein, B. Kostka, R. Rossmanith, M. Weisser, E. Steffens, G. Gerlach, and T. Baumbach, in Proceedings of the 10th European Particle Accelerator Conference, Edinburgh, Scotland, 2006, Ref. [4], pp. 3577-3579.

[17] Undulators, Wigglers and their Applications, Ref. [1], Vol. I, Chap. 5, pp. 182-190, ISBN 0-415-28040-0.

[18] J. Clarke, The Science and Technology of Undulators and Wigglers (Oxford Science Publication, New York, 2004), Chap. 9, pp. 171-176.

[19] S. Chouhan, R. Rossmanith, S. Strohmer, D. Doelling, A. Geisler, A. Hobl, and S. Kubsky, in Proceedings of the Particle Accelerator Conference, Portland, OR, 2003 (IEEE, New York, 2003), pp. 899-901.

[20] S. Prestemon, D. Dietderich, S. Bartlett, M. Coleman, S. Gourlay, A. Lietzke, S. Marks, S. Mattafirri, R. Scanlan, R. Schlueter, B. Wahrer, and B. Wang, IEEE Trans. Appl. Supercond. 15, 1236 (2005).

[21] D. Wollmann, Master's thesis, University of Technology Dresden, Germany, 2005.

[22] D. Doelling, A. Hobl, H.-U. Klein, D. Krischel, P. Komorowski, and M. Meyer-Reumers, in Proceedings of the 2007 Particle Accelerator Conference, Albuquerque, New Mexico, 2007 (IEEE, Albuquerque, New Mexico, 2007), pp. 935-937.

[23] D. Wollmann, A. Bernhard, P. Peiffer, R. Rossmanith, and T. Baumbach, in Proceedings of the 11th European Particle Accelerator Conference, Genua, Italy, 2008, pp. 2323-2325.

[24] J.D. Jackson, Classical Electrodynamics (John Wiley \& Sons, Inc., New York, 1999), Chap. 5, pp. 175-178, 3rd ed.

[25] I. Bronstein, K. Semendjajew, G. Musiol, and H. Muehlig, Taschenbuch der Mathematik (Verlag Harri Deutsch, Frankfurt am Main, 2001), Chap. 21.5, p. 1053, 5th ed. 